\title{
Carriage of colistin-resistant Gram-negative bacteria in children from communities in Cape Town (Tuberculosis child multidrug-resistant preventive therapy trial sub-study)
}

\section{Authors: \\ Yolandi Snyman ${ }^{1}$ Andrew C. Whitelaw ${ }^{1,2}$ Motlatji R.B. Maloba $a^{3,4}$ Anneke C. Hesseling ${ }^{5}$ Mae Newton-Foot ${ }^{1,2}$ (1) \\ Affiliations: \\ ${ }^{1}$ Department of Pathology, Faculty of Medicine and Health Sciences, Stellenbosch University, Cape Town, South Africa \\ ${ }^{2}$ National Health Laboratory Service, Tygerberg Hospital, Cape Town, South Africa \\ ${ }^{3}$ Department of Medical Microbiology, Faculty of Health Science, University of the Free State, Bloemfontein, South Africa \\ ${ }^{4}$ National Health Laboratory Service, Universitas Hospital, Bloemfontein, South Africa \\ ${ }^{5}$ Department of Paediatrics and Child Health, Faculty of Medicine and Health Sciences, Stellenbosch University, Cape Town, South Africa}

Research Project Registration Project Number: 3144

Corresponding author: Yolandi Snyman, 17770467@sun.ac.za

Dates:

Received: 26 Aug. 2020 Accepted: 10 Dec. 2020 Published: 10 Feb. 2021

Read online:

Scan this $Q R$ code with your smart phone or mobile device to read online.
Colistin is a last-resort antibiotic against multidrug-resistant, Gram-negative bacteria. Colistin resistance has been described in the clinical settings in South Africa. However, information on carriage of these bacteria in communities is limited. This study investigated gastrointestinal carriage of colistin-resistant Escherichia coli and Klebsiella spp. and mor genes in children from communities in Cape Town. Colistin-resistant E. coli was isolated from two participants $(4 \%, 2 / 50)$, and mcr-1-mcr-9 genes were not detected. Gastrointestinal carriage of colistinresistant Enterobacterales was rare; however, continuous extensive surveillance is necessary to determine the extent of carriage and its contribution to resistance observed in clinical settings.

Keywords: colistin resistance; Enterobacterales; children; healthy; communities; Cape Town; South Africa.

\section{Introduction}

Antibiotic resistance is threatening public health globally, and colistin remains one of the last-resort antibiotics for treating infections because of carbapenem-resistant Enterobacterales. Colistin resistance is increasingly being reported in Enterobacterales both worldwide and in South Africa, which is of great concern. ${ }^{1,2,3,4,5,6,7,8,9}$

The predominant mechanism of colistin resistance in Enterobacterales involves changes to the phosphate groups of lipid A by adding phosphoethanolamine (PEtN) and/or 4-amino-4-deoxy-L-arabinose (L-Ara4N), resulting in reduced anionic charge of the lipopolysaccharide (LPS). Mutations in the two-component regulatory systems, PhoPQ and $\mathrm{PmrAB}$, and inactivation of the $m g r B$ gene are the most common causes of colistin resistance. The mobile colistin resistance $(m c r-1)$ gene was first reported in 2016 in China. ${ }^{1}$ The $m c r$ gene encodes a PEtN transferase enzyme, which transfers a PEtN to lipid A, thereby conferring resistance to colistin. Additional plasmid-mediated $m c r$ genes, $m c r-2-m c r-10$ and different variants thereof, have since been detected, which also confer colistin resistance ${ }^{1,2} \mathrm{mcr}$ genes have been mostly isolated from Enterobacterales, especially Escherichia coli and Klebsiella pneumoniae, which are responsible for both nosocomial and community-acquired (CA) infections, including sepsis, pneumonia, urinary tract infections and intra-abdominal infections ${ }^{10,11}$; these organisms are also common gut commensal organisms. Numerous studies have detected the $m c r$ gene in isolates from clinical, community and environmental settings. 1,2,3,45,6

The $m c r-1$ gene has been identified in clinical isolates from at least 11 hospitals across South Africa, $, 7,9$ as well as in E. coli isolates from outpatients in Gauteng $(n=4)$ and the Western Cape $(n=1) .{ }^{8}$ The $m c r-1$ gene has also been detected in $E$. coli isolated from broiler chickens, a pig and final effluents from wastewater treatment plants in South Africa. ${ }^{3,4,5,6}$ However, there are limited data regarding the carriage of colistin-resistant organisms and $\mathrm{mcr}$ genes in healthy individuals from the community. The presence of $\mathrm{mcr}$ genes in the community could serve as a reservoir for colistin resistance in clinical settings, leading to difficult and more expensive treatment for human bacterial infections, increased hospitalisation, extended hospital stays and sometimes death. Gastrointestinal carriage of plasmid-mediated colistin resistance poses additional threat

How to cite this article: Snyman Y, Whitelaw AC, Maloba MRB, Hesseling AC, Newton-Foot M. Carriage of colistin-resistant Gram-negative bacteria in children from communities in Cape Town (Tuberculosis child multidrug-resistant preventive therapy trial sub-study). S Afr J Infect Dis. 2021;36(1), a241. https://doi.org/10.4102/sajid.v36i1.241

Copyright: @ 2021. The Authors. Licensee: AOSIS. This work is licensed under the Creative Commons Attribution License. 
as it may be transferred to other opportunistic pathogens in the gut. This study aimed to describe the gastrointestinal carriage of colistin-resistant organisms and $\mathrm{mor}$ genes in children from Cape Town communities.

\section{Methodology Study population}

Participants were enrolled in an ongoing Tuberculosis Child Multidrug-resistant Preventive Therapy (TB CHAMP) trial, a two-arm, cluster-randomised, double-blinded placebocontrolled phase- 3 trial evaluating the efficacy and safety of 6 months of levofloxacin preventive therapy for treating multidrug-resistant tuberculosis (MDR-TB), http://www.isrctn. com/ISRCTN92634082, in healthy children $<5$ years of age with household exposure to MDR-TB. The first 50 stool samples, collected between November 2017 and August 2018, were used. Children were enrolled from clinics based in Khayelitsha and Philippi in urban Cape Town, South Africa.

\section{Sample collection}

Stool specimens were collected by nurses from children who were enrolled in the trial before commencing study treatment using a standard operating procedure. Six scoops of formed stool or three scoops of loose stool were collected using a 2.5 millilitres $(\mathrm{mL})$ spoon (Lasec, South Africa) and mixed several times. Samples were delivered in a cooler box with an ice pack $\left(2^{\circ} \mathrm{C}-8^{\circ} \mathrm{C}\right)$ to the National Health Laboratory Services (NHLS) microbiology laboratory at Tygerberg Hospital and were stored at $-80^{\circ} \mathrm{C}$.

\section{Culture-based screening for colistin resistance}

Stool samples were cultured on a MacConkey agar (Sigma-Aldrich, South Africa) containing 10 milligrams per litre (mg/L) vancomycin (Sigma-Aldrich) and $2 \mathrm{mg} / \mathrm{L}$ colistin (Sigma-Aldrich) to isolate colistin-resistant E. coli and K. pneumoniae using the spread plate method. Distinct bacterial colonies were selected based on their morphological resemblance to E. coli or Klebsiella spp. on the MacConkey agar. Isolates were sub-cultured on a chromogenic UriSelect agar (NHLS Media Laboratory, Green Point, South Africa) for preliminary identification, and matrix-assisted laser desorption/ionisation time-of-flight mass spectrometry (MALDI-TOF; Bruker Daltonics, Bremen, Germany) was performed to confirm species identification.

Colistin resistance was confirmed by broth microdilution (BMD) following the European Committee on Antimicrobial Susceptibility Testing (EUCAST) guidelines and clinical breakpoints version 10 (minimum inhibitory concentration $[\mathrm{MIC}] \leq 2 \mathrm{mg} / \mathrm{L}$ : susceptible, MIC $>2 \mathrm{mg} / \mathrm{L}$ : resistant). Escherichia coli American Type Culture Collection (ATCC) 25922 and E. coli National Collection of Type Cultures (NCTC) 13846 (mcr-1 positive) were used as colistinsusceptible and -resistant control strains, respectively.

\section{Detection of mobile colistin resistance genes}

Genomic deoxyribonucleic acid (DNA) was extracted directly from $200 \mathrm{mg}$ of each stool sample using the $\mathrm{PSP}^{\circledR}$ Spin Stool DNA kit (Stratec Molecular, Germany) according to the manufacturer's instructions. Genomic DNA was extracted from colistin-resistant colonies using a crude boil-freeze extraction method. ${ }^{12}$ mcr-1 - mcr-5 genes were detected by polymerase chain reaction (PCR), as previously described. ${ }^{13}$ Singleplex PCR detection of the $m c r-6, m c r-7, m c r-8$ and $m c r-9$ genes was also performed using previously described primers, ${ }^{14}$ newly designed primers (MCR-9YF 5'-ATG CCT GTA CTT TTC AGG GTG AAA G-3' and MCR-9YR 5'-TTC CGC GAA TGC CGT GGC TAA-3') and previously described protocols.? No positive controls were available for $m c r-6-m c r-9$ genes.

\section{Ethical considerations}

Ethical approval was obtained from the Health Research Ethics Committee (HREC), Stellenbosch University for the main trial (M16/02/009) and this sub-study (S17/11/269).

\section{Results}

Bacterial growth was observed on the MacConkey agar for all 50 stool samples. A total of 70 isolates, 55 E. coli from 25 participants and $15 \mathrm{~K}$. pneumoniae from six participants, were obtained on the MacConkey agar with colistin and vancomycin. Escherichia coli and/or K. pneumoniae were obtained from the colistin containing media of 30 participants. More than one E. coli or Klebsiella spp. colony was isolated from some of the participants because of differences in colony morphology, and both E. coli and K. pneumoniae were isolated from one participant. For 20 of the participants, no E. coli or Klebsiella spp. were obtained from the colistin containing media. Colistin resistance was confirmed by BMD in only $3 \%(2 / 70)$ of the isolates, both of which were $E$. coli (MIC $=4 \mathrm{mg} / \mathrm{L})$. The colistin MICs of the remaining isolates were in the range of $0.125 \mathrm{mg} / \mathrm{L}-0.5 \mathrm{mg} / \mathrm{L}$. The two colistinresistant E. coli were from two separate individuals, and therefore, the carriage rates of colistin-resistant E. coli and K. pneumoniae were $4 \%(2 / 50)$ and $0 \%(0 / 50)$, respectively.

The plasmid-mediated $m c r-1$ to $m c r-9$ colistin resistance genes were not detected in either the colistin-resistant $E$. coli isolates or the DNA extracted directly from the stool samples.

\section{Discussion}

Colistin is regarded as one of the last-resort antibiotics, and therefore, any emerging resistance outside of hospital settings is of great concern. In South Africa, there is a lack of information regarding the carriage of colistin resistance and mechanisms of resistance in children and in the community, in general. In another Cape Town study, nasopharyngeal colonisation with resistant pathogens was reported in human immunodeficiency virus (HIV)-positive children, with extended-spectrum beta-lactamase production in $50 \%$ of Enterobacteriaceae ${ }^{15}$; this poses a risk of becoming infected by these resistant organisms. The TB CHAMP trial 
provided an opportunity to obtain data on gastrointestinal carriage of colistin resistance in healthy children from communities in Cape Town.

This study showed that carriage of colistin-resistant organisms was uncommon in healthy children enrolled from local communities, with $4 \%$ of participants carrying colistinresistant $E$. coli. The majority (68/70) of the colonies obtained on the colistin-containing media were not colistin-resistant based on BMD. This could be related to the poor diffusion of colistin in agar as previously reported ${ }^{16}$ or could be influenced by the amount of stool cultured, or the abundance of bacteria in the stool sample. However, resistance was confirmed by $\mathrm{BMD}$, which is considered the only reliable colistin susceptibility method. ${ }^{17}$ A previous study has selected colistin-resistant Gram-negative bacteria (GNB) with a higher $(4 \mathrm{mg} / \mathrm{L})$ colistin concentration in $\operatorname{agar}^{18}$; however, this approach has the potential to miss isolates with a borderlineresistant MIC (2 mg/L $-4 \mathrm{mg} / \mathrm{L})$. Development of a costeffective and user-friendly commercial BMD panel for colistin susceptibility testing or direct $\mathrm{mcr}$ screening could be valuable for surveillance purposes.

None of the $m c r-1-m c r-9$ genes were detected in any of the isolates or stool samples, suggesting that although $\mathrm{mor}$ genes have been detected in clinical settings in South Africa, ${ }^{7,9}$ these genes are not widely disseminated in these communities. The two colistin-resistant E. coli isolates could have chromosomal mutations in $p m r A, p m r B, p h o P, p h o Q$, ept $B$ or $m g r B$ genes, which lead to L-Ara $4 \mathrm{~N}$ and/or PEtN modification of lipid $\mathrm{A}$ in the bacterial outer membrane resulting in colistin resistance. ${ }^{19,20,21,22,23}$ In addition, other mor genes ( $m c r-10)$ or potentially undescribed, plasmidmediated colistin resistance genes could be responsible for colistin resistance.

To the best of our knowledge, this is the first study to investigate the carriage of colistin resistance in a South African community setting. Colistin resistance in communities, especially the dissemination of $\mathrm{mor}$ genes, could lead to infections with these resistant organisms, which could further spread to hospital settings. Although the sample size was small, our data suggest that community carriage of colistin-resistant organisms in children is uncommon. This also reassures that colistin can still be used in this community as a last-resort antibiotic when necessary. Ongoing surveillance is needed to better understand and estimate the prevalence of colistin resistance in the community.

\section{Conclusion}

We report a low carriage rate of colistin-resistant Enterobacterales and no plasmid-mediated colistin resistance genes in children sampled from the community level. Colistin is the last-resort antibiotic for the treatment of multidrugresistant GNB, and therefore, there is a need to understand the prevalence of colistin resistance in community settings. Ongoing surveillance is required in additional community settings in order to determine the extent of carriage and its contribution to resistance observed in clinical settings.

\section{Acknowledgements}

The authors would like to thank the entire TB CHAMP study team at DTTC for sampling. They also like to thank Chanté Brand, Remous Ocloo and Kristien Nel van Zyl for storing the samples and Chanté Brand for extracting the stool DNA.

\section{Competing interests}

The authors declare that they have no financial or personal relationships that may have inappropriately influenced them in writing this report.

\section{Authors' contributions}

Y.S. performed all experiments, interpreted and organised the project, and drafted the manuscript. M.N.F. and A.W. designed and supervised the study. M.N.-F., A.C.W., M.R.B.M. and A.C.H. interpreted the results, and edited the manuscript. All authors contributed to manuscript revision, read and approved the submitted version.

\section{Funding information}

The main trial was funded by the British Medical Research Council. This work is based on the research supported in part by the National Research Foundation of South Africa (Grant No. 118033) and the NHLS Research Trust. Y.S. is supported by the National Research Foundation of South Africa Innovation Masters Scholarship. A.C.H. is funded by a South African National Research Foundation SARCHi chair in Paediatric Tuberculosis.

\section{Data availability}

The authors confirmed that the data supporting the findings of this study are available within the research article.

\section{Disclaimer}

The views and opinions expressed in this article are those of the authors and do not necessarily reflect the official policy or position of any affiliated agency of the authors.

\section{References}

1. Liu Y-Y, Wang Y, Walsh TR, et al. Emergence of plasmid-mediated colistin resistance mechanism MCR-1 in animals and human beings in China: A microbiological and org/10.1016/S1473-3099(15)00424-7
ord

2. Wang $C$, Feng $Y$, Liu L, Wei L, Kang $M$, Zong Z. Identification of novel mobile colistin resistance gene mcr-10. Emerg Microbes Infect. 2020;9(1):508-516. https://doi. org/10.1080/22221751.2020.1732231

3. Mendelson M, Brink A, Gouws J, et al. The One Health stewardship of colistin as an antibiotic of last resort for human health in South Africa. Lancet Infect Dis. 2018;18(9):e288-e294. https://doi.org/10.1016/S1473-3099(18)30119-1

4. Igwaran A, Iweriebor BC, Okoh Al. Molecular characterization and antimicrobial resistance pattern of Escherichia coli recovered from wastewater treatment plants in Eastern Cape South Africa. Int J Environ Res Public Health. 2018;15(6):1237. https://doi.org/10.3390/ijerph15061237

5. Perreten V, Strauss C, Collaud A, Gerber D. Colistin resistance gene mcr-1 in Avianpathogenic Escherichia coli in South Africa. Antimicrob Agents Chemother. 2016;60(7):4414-4415. https://doi.org/10.1128/AAC.00548-16 
6. Founou LL, Founou RC, Allam M, Ismail A, Essack SY. Extended-spectrum betalactamase-producing Escherichia coli harbouring mcr-1 gene isolated from pigs in
South Africa. S Afr Med J. 2018;108(10):796-797. https://doi.org/10.7196/ South Africa. S Afr Med
SAMJ.2018.v108i10.13508

7. Newton-Foot $M$, Snyman $Y$, Maloba MRB, Whitelaw AC. Plasmid-mediated mcr-1 colistin resistance in Escherichia coli and Klebsiella spp. clinical isolates from the Western Cape region of South Africa. Antimicrob Resist Infect Control. 2017;6:78. https://doi.org/10.1186/s13756-017-0234-8

8. Coetzee J, Corcoran C, Prentice E, et al. Emergence of plasmid-mediated colistin resistance (MCR-1) among Escherichia coli isolated from South African patients. S Afr Med J. 2016;106(5):35-36. https://doi.org/10.7196/SAMJ.2016.v106i5.10710

9. Poirel L, Kieffer N, Brink A, Coetze J, Jayol A, Nordmann P. Genetic features of MCR-1-producing colistin-resistant Escherichia coli isolates in South Africa. Antimicro Agents Chemother. 2016;60(7):4394-4397. https://doi.org/10.1128/AAC.00444-16

10. Williams PCM, Isaacs D, Berkley JA. Antimicrobial resistance among children in sub-Saharan Africa. Lancet Infect Dis. 2018;18(2):e33-e44. https://doi. org/10.1016/S1473-3099(17)30467-X

11. Falagas ME, Kasiakou SK. Colistin: The revival of polymyxins for the management of multidrug-resistant Gram-negative bacterial infections. Clin Infect Dis. 2005;40(9):1333-1341. https://doi.org/10.1086/429323

12. Ndlovu T, Le Roux M, Khan W, Khan S. Co-detection of virulent Escherichia col genes in surface water sources. PLoS One. 2015;10(2):e0116808. https://doi. org/10.1371/journal.pone.0116808

13. Snyman Y, Whitelaw AC, Reuter S, Dramowski A, Maloba MRB, Newton-Foot M. Clonal expansion of colistin-resistant Acinetobacter baumannii isolates in Cape Town, South Africa. Int J Infect Dis. 2020;91:94-100. https://doi.org/10.1016/j.ijid.2019.11.021

14. Wang $X$, Wang $Y$, Zhou $Y$, et al. Emergence of a novel mobile colistin resistance gene, mcr-8, in NDM-producing Klebsiella pneumoniae. Emerg Microbes Infect. 2018;7(1):122. https://doi.org/10.1038/s41426-018-0124-z

15. Cotton MF, Wasserman E, Smit J, Whitelaw A, Zar HJ. High incidence of antimicrobial resistant organisms including extended spectrum beta-lactamase producing Enterobacteriaceae and methicillin-resistant Staphylococcus aureus in nasopharyngeal and blood isolates of HIV-infected children from South. BMC Infect Dis. 2008;8:1-10. https://doi.org/10.1186/1471-2334-8-40
16. Matuschek E, Åhman J, Webster C, Kahlmeter G. Antimicrobial susceptibility testing of colistin - Evaluation of seven commercial MIC products against standard broth microdilution for Escherichia coli, Klebsiella pneumoniae, Pseudomonas aeruginosa, and Acinetobacter spp. Clin Microbiol Infect. 2018;24(8):865-870. https://doi.org/10.1016/j.cmi.2017.11.020

17. EUCAST. Recommendations for MIC determination of colistin (polymyxin E) as recommended by the joint CLSI-EUCAST polymyxin breakpoints working group [homepage on the Internet]. EUCAST; 2016 [cited n.d.]. Available from: http://www.eucast.org/fileadmin/src/media/PDFs/EUCAST_files/General_ documents/Recommendations_for_MIC_determination_of_colistin March_2016.pdf

18. Zurfluh K, Stephan R, Widmer A, et al. Screening for fecal carriage of MCR producing Enterobacteriaceae in healthy humans and primary care patients. Antimicrob Resist Infect Control. 2017;6(1):7-10. https://doi.org/10.1186/ s13756-017-0186-z

19. Olaitan AO, Morand S, Rolain J-M. Mechanisms of polymyxin resistance: Acquired and intrinsic resistance in bacteria. Front Microbiol. 2014;5:643. https://doi. org/10.3389/fmicb.2014.00643

20. Wright MS, Suzuki Y, Jones MB, et al. Genomic and transcriptomic analyses of colistin-resistant clinical isolates of Klebsiella pneumoniae reveal multiple pathways of resistance. Antimicrob Agents Chemother. 2015;59(1):536-543. https://doi.org/10.1128/AAC.04037-14

21. Cannatelli A, Giani T, D'Andrea MM, et al. MgrB inactivation is a common mechanism of colistin resistance in KPC-producing Klebsiella pneumoniae of clinical origin. Antimicrob Agents Chemother. 2014;58(10):5696-5703. https:// doi.org/10.1128/AAC.03110-14

22. Lippa AM, Goulian M. Feedback inhibition in the PhoQ/PhoP signaling system by a membrane peptide. PLoS Genet. 2009;5(12):e1000788. https://doi. org/10.1371/journal.pgen.1000788

23. Poirel L, Aurélie J, Nordmann P. Polymyxins: Antibacterial activity, susceptibility testing and resistance mechanisms encoded by plasmids or chromosomes. Clin Microbiol Rev. 2017;30(2):557-596. https://doi.org/ 10.1128/CMR.00064-16 